\title{
Companion detection limits with adaptive optics coronagraphy
}

Ben R. Oppenheimer, Richard G. Dekany, Thomas L. Hayward, Bernhard Rainer Brandl, Mitchell Troy, et al.

Ben R. Oppenheimer, Richard G. Dekany, Thomas L. Hayward, Bernhard Rainer Brandl, Mitchell Troy, Eric E. Bloemhof, "Companion detection limits with adaptive optics coronagraphy," Proc. SPIE 4007, Adaptive Optical Systems Technology, (7 July 2000); doi: 10.1117/12.390385 


\title{
Companion Detection Limits with Adaptive Optics Coronagraphy
}

\author{
B. R. Oppenheimer, ${ }^{a}$ R. G. Dekany, ${ }^{b}$ T. Hayward, ${ }^{c}$ \\ B. Brandl, ${ }^{c}$ M. Troy ${ }^{b}$ and E. E. Bloemhof \\ ${ }^{a}$ Department of Astronomy, University of California-Berkeley, \\ 601 Campbell Hall, Berkeley, CA 94720, USA \\ ${ }^{b}$ Jet Propulsion Laboratory, Oak Grove Drive, Pasadena, CA 91125, USA \\ ${ }^{c}$ Department of Astronomy, Cornell University, Ithaca, NY 11410, USA \\ ${ }^{d}$ Palomar Observatory, California Inst. of Technology, Pasadena, CA 91125 USA
}

\begin{abstract}
We present a detailed observational study of the capabilities of the Palomar Adaptive Optics System and the PHARO near infrared camera in coronagraphic mode. The camera provides two different focal plane occulting masks consisting of completely opaque circular disks of diameter 0.433 arcsec and 0.965 arcsec, both within the cryogenic dewar. In addition, three different pupil plane apodizing masks (a. k. a. Lyot masks) are provided which downsize the beam. The six different combinations of Lyot mask and focal plane mask provide for different levels of suppresion of the point spread function of a bright star centered on the focal plane mask. We obtained images of the bright nearby star Gliese 614 with all six different configurations in the K-band filter. Herein, we provide an analysis of the dynamic range acheivable with these configurations. The dynamic range (the ratio of the primary star intensity to the intensity of the faintest point source detectable in the images) is a complicated function of not only the angular separation of the primary star and companion, but also of the azimuthal angle because of the complex point spread fucntion of the primary star, which is also wavelength dependent. However, beyond 2.5 arcseconds from the star, regardless of the wavelength of the observation, the detection limit of a companion is simply the limiting magnitude of the image, as determined by the sensitivity of the PHARO camera. Within that radius, the dynamic range is at least 8 magnitudes at the $5 \sigma$ level and as high as 12 in a one second exposure. This represents a substantial gain over similar techniques without adaptive optics, which are generally limited to radii beyond two arcsec. We provide a quantitative discussion and recommendation for the optimal configuration along with a detailed comparison with recent theoretical predictions of $\mathrm{AO}$ coronagraphic performance.
\end{abstract}

Keywords: Adaptive Optics, Coronagraphy, High Dynamic Range Imaging

\section{INTRODUCTION}

In 1939, M. Bernard Lyot addressed the Royal Astronomical Society. ${ }^{1}$ He described what he called a coronagraph, an optical system fitted to a telescope on the Pic du Midi, which permitted him to make photographs and spectra of the sun's corona. Previously, the sun's corona could only be observed successfully during total eclipses, because the corona is about a million times fainter than the sun's disk, even an arcminute away. Today, the same sort of device is used to produce extremely high dynamic range images in the immediate vicinity of stars on the sky. In this case, the target is a point source and the interest is in detecting either faint companions or dust disks and shells surrounding the central star. The principles of the coronagraph, however, remain the same.

The basic idea behind a coronagraph is that a focal plane stop is introduced with approximately the size of the stellar image. This blocks out most of the light of the star. However, light is scattered in the atmosphere and in the telescope optics, so a pupil plane stop is introduced as well, to reduce the broad halo of light from the star. A detailed explanation of this, from the point of view of fourier optics is presented in several other publications. ${ }^{2,3}$ Many examples of the usefulness of stellar coronagraphy already exist, some which employ tip-tilt wavefront correction. ${ }^{4-7}$

Further author information: (Send correspondence to B. R. O.)

E-mail: bro@astro.berkeley.edu.

T. Hayward is now at Gemini Observatory, 670 N. A'ohoku Place, Hilo, HI 96720 USA. 
With the advent of adaptive optics, it is now possible to achieve high dynamic ranges at extremely small separations from a given star, because we can now design coronagraphs that are optimized for the diffraction limit. The adaptive optics system provides a nearly diffraction limited image (fractions of an arcsecond in width) on top of a seeing-disk halo. A coronagraph, fitted behind an adaptive optics system, therefore, can permit one to probe the vicinities of nearby stars on the spatial scale of the solar system. With this in mind, the Palomar High Angular Resolution Observer (PHARO) infrared camera, which is the principal instrument for the Palomar Adaptive Optics System $^{8}$ (PALAO), was designed with a coronagraphic imaging mode.

Theoretical calculations ${ }^{2}$ show that the optimal size of the focal plane spot is between 4 and 9 times $\lambda / D$, where $\lambda$ is the wavelength of observation and $D$ is the diameter of the telescope aperture. These theoretical calculations also show that a careful balance between dynamic range and throughput must be achieved when designing the coronagraph. The throughput is reduced by the size of the pupil plane stop, which principally downsizes the beam. In Ref. 2, this optimal balance is shown to require that if the focal plane stop is $s \lambda / D$ then the pupil plane stop should mask out a border of the pupil that is $\mathrm{F} D / s$ wide, where $\mathrm{F}$ is a value betweeb 0.25 and 0.50 depending upon the quality of the adaptive optics correction. So for a focal plane stop of $9 \lambda / D$, the Lyot mask should downsize the pupil to between 90 and $80 \%$ of its initial width.

We have conducted observations of the star Gliese 614 (HD 145675, BD+44 2549, a $V=6.67$, K0V star at 18.14 pc with no known companions or surrounding diffuse emission) in the $\mathrm{K}$ band using PHARO and PALAO in order to assess experimentally the dynamic range possible with this instrument.

\section{PHARO INSTRUMENT SETTINGS}

The coronagraph on PHARO permits six different configurations: there are two different focal plane stops and three different pupil plane stops (a. k. a. Lyot masks). The focal plane masks are 0.433 " and 0.965 " in diameter. This corresponds to 9 and 19 times the diffraction limit of the Palomar $5 \mathrm{~m}$ telescope at $\mathrm{J}$ band, or 4 and 9 times the diffraction limit at $\mathrm{K}$ band. These focal plane masks are hard-edged. Masks with gaussian transmission functions have been shown to be superior, but are difficult to manufacture.

In PHARO, the three Lyot masks downsize the pupil to $100 \%$ (the standard imaging mask), $290 \%$ (the "medium" Lyot mask) and $\sim 80 \%$ (the "big" mask). The pupil masks in PHARO not only mask out the outer part of the pupil but also restrict the inner part, essentially covering the secondary obscuration. For example, the big mask reduces both the inner and outer radius of the annular pupil by $1 / 5$.

\section{OBSERVATIONS}

Observations of the star Gliese 614 were made on 1999 July 30 at approximately 6:00 UT. PALAO used the star as a guide and was able to run at a closed loop frequency of $550 \mathrm{~Hz}$, with the fast steering mirror operating at $100 \mathrm{~Hz}$. The K-band images taken before placing the star under the focal plane mask showed a clearly diffraction-limited core ( 0.11 ") and the measured Strehl ratio was approximately $50 \%$. The uncorrected seeing in the K band was 1.1 ". The loop maintained a stable lock on the star throughout observations which lasted for over one hour, and measurements of the point spread function after the observations indicated a $50 \%$ Strehl ratio as well. If the Strehl ratio were significantly improved (i. e. $>80 \%$ ), the dynamic range achieved would be far superior. We show in the following sections that the ultimate limiting factor is how much light remains in the stellar halo.

Observations were carried out in the following manner. First we observed Gliese 614 with the smaller 0.433 " focal plane mask. We placed the star under the mask by moving the SSMs (see Ref. 8) after closing the AO loop on the star. In three separate sets of images we used the standard, medium and big Lyot masks. These sets of images consisted of ten two second exposures, ten two second exposures and three ten second exposures for each of the settings respectively. The pixel scale on PHARO was set to 25 mas in order to sample the point spread function as thoroughly as possible. A second set of images were taken with the 0.965 " focal plane mask in place. Again, each of the three Lyot masks were used and the images collected were nine two second exposures, nine two second exposures and nine twenty second exposures for the standard, medium and big Lyot masks respectively. These exposure times were chosen so that no part of the image would be saturated by the starlight. In all cases, accompanying sky frames were taken immediately after each data set. These were obtained by opening the AO loop and nodding the telescope by $50 " \mathrm{~N}$, a blank field for this instrument at these exposure times. 
Additional exposures of the star, unocculted by the focal plane mask were obtained at each of the settings. This permitted us to measure photometry relative to Gliese 614 in the field. In order not to saturate the detector in these images, we had to insert a $0.1 \%$ neutral density filter. This filter does affect the point spread function of the star, but only on the $.5 \%$ level, so its impact on the relative photometry was minimal. To check this, we used the known $\mathrm{K}$ band magnitude of Gliese 614 and measured the transmission of the filter to be $0.98 \%$. The photometric exposures consisted of six two second exposures for the standard Lyot mask, two ten second exposures through the medium mask and two ten second exposures through the big mask.

\section{DATA REDUCTION}

We reduced the data using the IRAF package of software from NOAO. The data were sky subtracted, flatfielded (using sky flats obtained at the beginning of the night), summed without any pixel shift, and cosmic rays were removed with an iterative algorithm that finds single pixel deviations greater than $4 \sigma$ from the surrounding mean. We did not use any pixel shift because it was unnecessary. The reacquisition of the AO loop lock after sky frames was extremely good so that the star was kept at the same pixel location to an accuracy of better than 0.1 pixels. The photometry frames were reduced in the same manner.

After the reduction, we normalized all of the integration times to one second. This permits logical comparison of the various settings $(\S 5)$.

\section{ASSESSMENT OF DYNAMIC RANGE}

In order to assess the dynamic range of the images, we used a technique previously outlined. ${ }^{9}$ This essentially involves the introduction of artificial point sources at various sample radial distances from the central, obscured star. The goal of these measurements is to produce the $5 \sigma$ detection limits as a function of radius from the star. The detection limits are specified as the magnitude difference between the star and the putative companion. Near the star, the star light is the limiting factor, providing a bright background and increasing the noise in that region. Beyond about 2 ", the star is relatively unimportant and the instrument detection limits determine the maximum possible dynamic range.

Artificial point sources were introduced as Airy functions, with added photon noise and read noise matching the detector characteristics, and the integration time was set to one second. Because we are dealing with very faint objects in the image (near the detection limit), only the central core of these artificial sources is visible. In reality, the point spread functions of point sources that are off axis and reimaged by a coronagraph will have somewhat more complex point spread functions. ${ }^{3}$ However, we are really only concerned with the peak of the companion point spread function, the low-level details being obscured by the noise from the sky and the detector read out electronics.

The brightness of the artificial point sources was set so that a star of the same magnitude as Gliese 614 would have an instrumental magnitude of 0 . Thus the artificial point source magnitudes give the dynamic range directly.

In order to increase the accuracy of the measurements, artificial stars were introduced along a radial line at five different azimuthal angles $\left(0,18,45,67\right.$ and $90^{\circ}$ right of up on the detector). Part of the reason for doing this is that the halo of light from the central star is not axially symmetric, though it does exhibit four-fold symmetry about the star. Fig. 1 shows a representative image of this type. By averaging the detection limits for each of the azimuthal angles used, we arrive at an average dynamic range function.

Fig. 2 shows the azimuthally averaged dynamic range functions for the six possible settings and an integration time of 1 second. One second is chosen to allow for ease in modifying the curves by integration time. To adjust the curve for a given exposure time, $t$, simply shift the y-axis $(\Delta \mathrm{mag})$ by adding $2.5 \log \left(t^{1 / 2}\right)$, where $t$ is in seconds. In the large radius limit, where the dynamic range curve levels off, the detector limit is reached. This part of the curve will simply follow the immediately preceding part (at lower radius) in a linear fashion for deeper exposure times.

There are several important features of Fig. 2 to note. The larger mask and larger Lyot stop provide superior results by up to a magnitude. In addition, it should be noted that both figures demonstrate the strong effect of the Lyot stop. In Fig. 2a the occulting mask and the Lyot stop are not well matched to the instrument. As a result, the big Lyot stop simply causes the telescope to behave as if it had a smaller pupil, with poorer detection limits at all radii. The medium stop inreases the dynamic range at small radii, but eventually matches the effect of the standard Lyot stop. In Fig. 2b, The big Lyot stop clearly limits the ultimate detection limit of the image in the large radius regime, because it downsizes the telescope aperture. However, at low radii, it provides superior suppression of the 


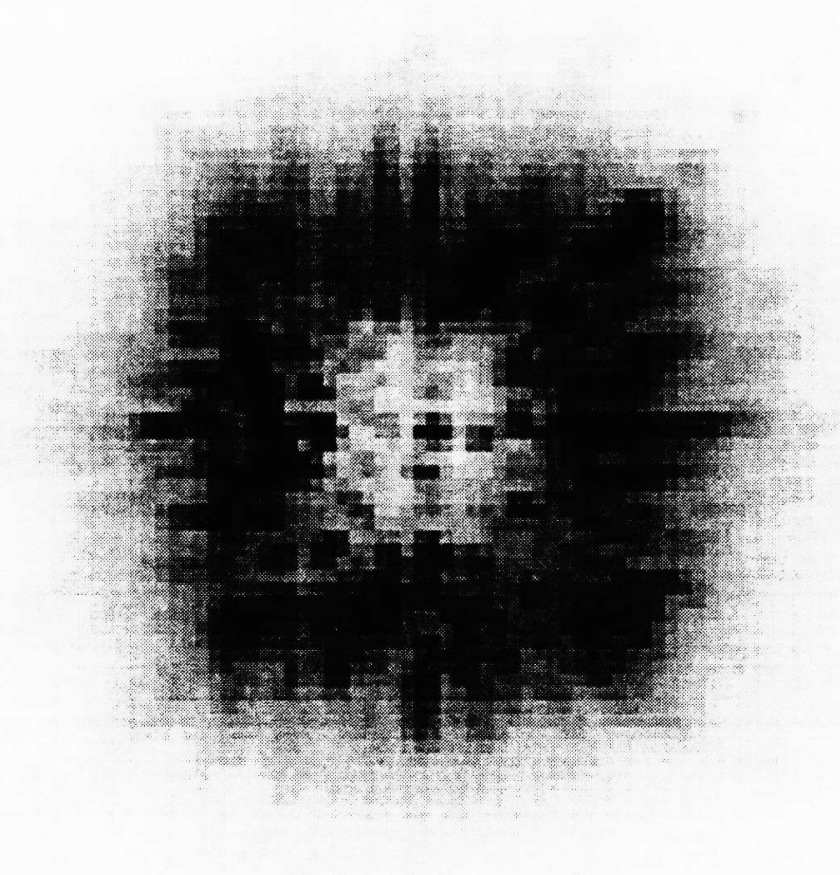

Figure 1. A $256 \times 256$ pixel portion of the reduced K-band image of Gliese 614 with the 0.965 " focal plane mask $(\sim 9 \lambda / D)$ and the big Lyot stop (with the inner diameter expanded by $1 / 5$ and the outer diameter reduced by $1 / 5)$. The pixel scale is 25 mas and total exposure time is 180s. Artificial point sources have been introduced as Airy functions along the radius $45^{\circ}$ right of up. These artificial sources are introduced at the $5 \sigma$ detection level and are used to produce the dynamic range functions in $\$ 5$. 

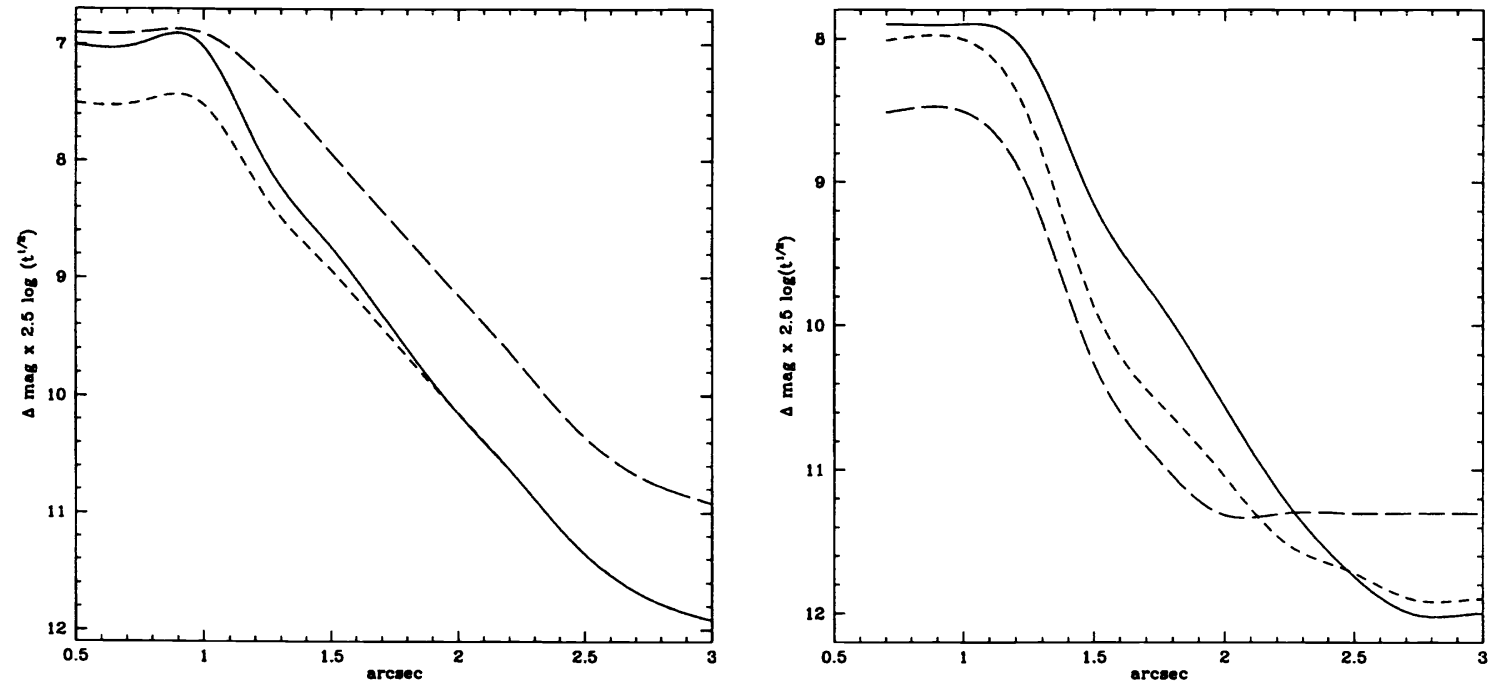

Figure 2. $5 \sigma$ detection limits for faint point sources near bright stars in K band, (a, left) with the 0.433 " focal plane mask and (b, right) with the 0.965 " mask. The three curves correspond to the standard Lyot stop (solid line), the medium Lyot stop (short dashed line) and the big Lyot stop (long dashed line). The curve is presented in terms of magnitude difference from a bright star, in this case a star with $\mathrm{V}=6.67, \mathrm{~K}=4.71$, for an integration time of 1 second. The ordinate indicates the dynamic range as a function of integration time, $t$, in seconds.

central star's point spread function. Thus, the curve corresponding to the big Lyot stop in Fig. $2 \mathrm{~b}$ starts out lower (higher dynamic range) than the others, but ultimately crosses the medium and standard Lyot stop curves. This clearly demonstrates that one must carefully choose the proper setting depending upon what radius is of interest to the observing program, and that the optical matching of the Lyot stop and focal plane mask is absolutely critical.

The big Lyot stop also provides a much faster fall-off of the central star point spread function. In Fig. $2 \mathrm{~b}$ the slope of the curves is much steeper than in Fig. 2a. We note again that, in $\mathrm{K}$ band, the 0.965 " mask is about 9 times the diffraction limit and the $\mathbf{0 . 4 3 3 " ~ m a s k ~ i s ~} 4$ times the diffraction limit. The theoretical calculations mentioned above predicted that a mask larger than $4 \lambda / D$ would be best. There is general agreement with these calculations in these curves. According to those calculations, ${ }^{2}$ the curves in Fig. 2a should approach those of Fig. $2 \mathrm{~b}$ as one shortens the observing wavelength. It follows that in $\mathrm{J}$ band, where the 0.433 " mask is $9 \lambda / D$, that the medium Lyot stop and the 0.433 " mask will provide results comparable to the 0.965 " mask and the big Lyot stop in the $\mathrm{K}$ band at similar Strehl ratios.

One interesting feature in the dynamic range curves is the leveling off below the one arcsecond radius. This is due to the apparent "plateau" in the point spread function inside the waffle pattern. ${ }^{10}$ While in most cases this plateau is not important, it is the dominant factor in these high dynamic range situations. Furthermore, the bump at nearly one arcsec, most clearly seen in the curve corresponding to the $\mathbf{0 . 4 3 3}$ " mask and the standard Lyot stop, is a direct manifestation of the waffle pattern.

The conclusions and results just described are easily understood if one considers the coronagraph from the point of view of Fourier optics. After the focal plane mask, the pupil is reimaged (a Fourier transform of the focal plane). The unocculted light from the central star is now largely concentrated at the edge of the pupil, the high frequency part of the pupil plane (low spatial frequency in the focal plane). The Lyot stop acts as a low-pass filter in the pupil plane by blocking out this light. As a result, the low spatial-frequency component in the focal plane is subdued. In other words, the Lyot stop acts to suppress the broad wings of the point spread function. The effect is clearly seen in Fig. $2 \mathrm{~b}$ where the big Lyot stop provides a clear improvement over the standard one. (n. b. the standard Lyot 
stop in these plots is essentially equivalent to direct imaging without the coronagraph, since the coronagraph is only acting to suppress the peak of the stellar image.)

We must note that the central star used in these cases was $\mathrm{K} 0 \mathrm{~V}$ star with $V=6.67$. If a fainter star is used, the same curves will apply, but the limiting magnitude (level part of the curve) will be lowered to limit the dynamic range at smaller radii. For example, if the limiting magnitude of the image is smaller than the central star magnitude plus 8 , the image's limiting magnitude will be the dominant effect at all radii.

\section{OBSERVING RECOMMENDATIONS}

The curves presented in $\S 5$ can be used as a point of reference for designing an observing program seeking faint light sources around bright stars. For imaging from 0.6 to 2.5 " radii, the maximum dynamic range is achieved with the 0.965 " focal plane mask and the big Lyot stop in $\mathrm{K}$ band and the 0.433 " mask and medium Lyot stop in J band. Outside 2.5", it is optimal to use the standard Lyot mask to maximize the limiting magnitude of the image and the 0.965 " focal plane mask to minimize the central star light. We summarize these recommendations in Table 1.

Table 1. Settings to Achieve Maximum Dynamic Range for Radii $<2.5 \%, 50 \%$ Strehl

\begin{tabular}{|c|c|c|c|}
\hline & J Band & H Band & K Band \\
\hline \hline Focal Plane Mask & $0.433 "$ & $0.965 "$ & $0.965 "$ \\
\hline Lyot Stop & Medium & Medium & Big \\
\hline
\end{tabular}

In addition to these setting recommendations, we strongly encourage observers to use multiple Cassegrain ring rotation angles. By rotating the point spread function on the sky one eliminates the problem of confusing a putative companion with an artifact of the central star's point spread function. This is a particularly serious effect in high dynamic range imaging. If rotation of the instrument cannot be performed (it requires a fairly substantial loss of observing time), then significantly lower dynamic range should be considered as the detection limit. We note that Fig. 1, a 180s exposure, shows that many of the knots in the broad part of the point spread function are quite long-lived. In fact those knots are probably the higher order Zernike terms of the calibration point spread function of the AO system. In the calibration phase, when the centroid offsets are determined, to which the AO system will drive the light of the guide star, only the first 10 Zernikes are corrected at present.

\subsection{Spectroscopic Observations}

The most intriguing motivation for finding companions of nearby, bright stars is in the spectroscopic study of them, whether they be low-mass stars, brown dwarfs or ultimately planets. We have attempted spectroscopic observations with PHARO and PALAO of some very close binaries with varying degrees of dynamic range. It has become clear through these studies that the magnitude difference for spectroscopic observations must be substantially smaller than for direct imaging. The principal difficulty is due to contamination of the companion's spectrum by the light in the broad halo of the AO point spread function. Furthermore, the PHARO and PALAO combination does not permit the use of the coronagraph and the spectrograph simultaneously. Effectively this means that the central star is contributing far more "background" light in spectroscopic mode than in coronagraphic imaging mode. The ideal situation would be to design the spectrograph to follow the coronagraph in the light path. Barring this, one should place the slit of the spectrograph so that the companion is centered and at least a comparable amount of the central star's light passes through the slit. These two spectra should be well-separated, so that individual spectra of the central star and the companion can be obtained. The level of contamination can then be assessed by measuring the light level immediately around the companion signal in the slit. By obtaining both spectra, the spectrum of the central star can then be properly subtracted from the companion spectrum. We note that this is contrary to standard practice where one would place the slit perpendicular to the line connecting the star and companion in an effort to minimize the contamination. This makes the removal of the central star spectrum prohibitive.

\subsection{Acknowledgments}

BRO acknowledges the support of a Hubble Space Telescope Postdoctoral Fellowship and the support of FUTDI. We thank Anand Sivaramakrishnan for useful comments and a careful reading of the manuscript. 


\section{REFERENCES}

1. M. B. Lyot, "A study of the solar corona and prominences without eclipses," Mon. Not. Royal Astron. Soc. 99, pp. 580-594, 1939.

2. A. Sivaramakrishnan, C. D. Koresko, R. B. Makidon, T. Berkefeld, and M. J. Kuchner, "Ground-based coronagraphy with high order adaptive optics," Astrophys. J. in prep., 2000.

3. F. Malbet, "High angular resolution coronagraphy for adaptive optics," Astron. Astrophys. Suppl. Ser. 115, pp. 161-174, 1996.

4. D. A. Golimowski, M. Clampin, S. T. Durrance, and R. H. Barkhouser, "High resolution ground-based coronagraphy using image-motion compensation," Appl. Opt. 31, pp. 4405-4416, 1992.

5. D. A. Golimowski, S. T. Durrance, and M. Clampin, "Coronagraphic imaging of the $\beta$ Pictoris circumstellar disk," Astrophys. J. Lett. 411, pp. L41-L44, 1993.

6. T. Nakajima, S. T. Durrance, D. A. Golimowski, and S. R. Kulkarni, "Coronagraphic search for brown dwarfs around nearby stars," Astrophys. J. 428, pp. 797-804, 1994.

7. T. Nakajima and D. A. Golimowski, "Coronagraphic imaging of the pre-main-sequence stars: remnant envelopes of star formation seen in reflection," Astron. J. 109, pp. 1181-1198, 1995.

8. R. G. Dekany, G. Brack, D. Palmer, B. R. Oppenheimer, T. L. Hayward, and B. Brandl, "First tip-tilt correction with the palomar 200-in. adaptive optics system," Proc. SPIE 3353, pp. 56-59, 1998.

9. B. R. Oppenheimer, Direct detection of brown dwarf companions of nearby stars, California Institute of Technology, Pasadena, CA, 1999.

10. E. E. Bloemhof, K. A. March, R. G. Dekany, M. Troy, and B. R. Oppenheimer, "Stability of the adaptive optics point spread function: metrics, deconvolution and initial Palomar results," Proc. SPIE this volume, 2000. 